Original Study

\title{
Relationship of Patient Volume and Service Concentration With Outcome in Geriatric Rehabilitation
}

\author{
Marije S. Holstege MSc ${ }^{a, b, *}$, Ineke G. Zekveld MSc ${ }^{a, c}$, Monique A.A. Caljouw MSc ${ }^{a}$, \\ Peter Bob Peerenboom MD, $\mathrm{PhD}^{\mathrm{c}}$, Romke van Balen MD, PhD ${ }^{\mathrm{a}}$, Jacobijn Gussekloo MD, PhD ${ }^{\mathrm{a}}$, \\ Wilco P. Achterberg MD, PhD ${ }^{\mathrm{a}}$ \\ ${ }^{a}$ Department of Public Health and Primary Care, Leiden University Medical Center, Leiden, The Netherlands \\ ${ }^{\mathrm{b}}$ Department of Innovation and Development, Evean, Purmerend, The Netherlands \\ ${ }^{\mathrm{c}}$ Tangram Health Care Consultancy, Elst, The Netherlands
}

\section{Keywords:}

Geriatric rehabilitation

postacute care

length of stay

discharge destination

\begin{abstract}
A B S T R A C T
Objective: Although geriatric rehabilitation (GR) is beneficial for restoration of activities and participation after hospitalization of vulnerable older persons, little is known about the optimal organization of care of these postacute facilities. This study examines the relationship of patient volume and service concentration with successful GR (short length of stay and discharge home) in skilled nursing facilities (SNFs). Design: A national multicenter retrospective cohort study.

Setting and participants: All patients indicated for GR in a Dutch SNF.

Measurements: Nurses filled out digital registration forms from patient records. Patients were studied in 3 predefined diagnostic groups: total joint replacement, traumatic injuries, and stroke. Facility characteristics were obtained by structured telephone interviews with facility managers. Volume was based on the number of discharges in a 3-month period and categorized in low-, medium-, and high-volume facilities. Concentration was defined at the organizational level in which the population consists of $80 \%$ or more of 1 or 2 diagnostic groups, with the prerequisite of having a minimum of 10 rehabilitation beds.

Results: From 88 facilities, 2269 GR patients (mean age 78.2 years [SD 9.7]; $68.2 \%$ female) were included. The median length of stay in the SNF was 45 days (interquartile range $23-81$ ), $57 \%$ of the patients were discharged home, and 9.8\% died during GR. Of patients with total joint replacement $(\mathrm{n}=501)$, concentration was related to successful rehabilitation (odds ratio 5.7 ; 95\% confidence interval $1.3-24.3$; $P=.020$, adjusted for age and gender); this relationship was not found for patients with traumatic injuries or stroke. Volume showed no relation with successful rehabilitation in any of the 3 diagnostic groups.

Conclusion: This study may indicate that concentration in an SNF, as a proxy for specialization, favors successful GR in total joint replacement. This relationship was not found for the traumatic injuries or stroke groups, or for volume. The relation on functional outcome in GR needs further investigation.
\end{abstract}

Copyright (c) 2013 - American Medical Directors Association, Inc.
With the increasing population of vulnerable older persons, the number of hospital admissions will increase worldwide. ${ }^{1}$ Patients in acute medical care with multiple geriatric conditions are at high risk for functional decline and institutionalization. Geriatric rehabilitation

This study was funded by the Health Care Insurance Board (CVZ, The Netherlands).

The authors declare no conflicts of interest.

* Address correspondence to Marije S. Holstege, MSc, Department of Public Health and Primary Care, Leiden University Medical Center, PO Box 9600, 2300 RC Leiden, The Netherlands.

E-mail address: m.s.holstege@lumc.nl (M.S. Holstege).
(GR) in a postacute care (PAC) setting is beneficial for restoration of activities and participation. ${ }^{2-4} \mathrm{GR}$ is defined as a multidisciplinary set of evaluative, diagnostic, and therapeutic interventions with the purpose to restore functioning or enhance residual functional capability in older people with disabling impairments. ${ }^{5}$

In hospital care, a higher volume of complex high-risk treatments is recognized as an indicator for better outcomes. ${ }^{6,7}$ However, in a PAC setting, such as a skilled nursing facility (SNF), the relation between the organization of GR and outcomes have rarely been studied. ${ }^{8-11}$ Among inpatient rehabilitation facilities, stroke units have proven efficient, although high-quality randomized clinical trials (RCTs) are still lacking for units specifically designed for the care of geriatric 
patients. ${ }^{11-13}$ In addition, Li et al $^{8}$ found that a higher patient volume had a positive effect on reducing the risk of rehospitalization of patients admitted for PAC to an SNF. Tian et al $^{14}$ reported that patients receiving joint replacements had the most efficient treatment in a medium-volume SNF (ie, 100-183 admissions per annum) and inpatient rehabilitation facilities.

In the Netherlands, GR includes personal care, nursing, accommodation, counseling, and treatment in an SNF. The rehabilitation treatment has an interdisciplinary patient-centered approach that requires a temporary residence situation (on average 2 months) with a therapeutic living environment. Each client is reimbursed for an average of 18 to 22 hours per week of nursing care and 4 hours per week of treatment. The rehabilitation activities are performed by a multidisciplinary team led by an elderly care physician (ie, a medical practitioner who has specialized as a primary care expert in geriatric medicine and qualified as a basic specialist with expertise in geriatric medicine). ${ }^{15}$ The multidisciplinary team involves nursing staff, physiotherapists, psychomotor therapists, occupational therapists, psychologists, social workers, speech therapists, dieticians, and pharmacists. They coordinate their work with regular team meetings in which rehabilitation care plans are evaluated.

Anticipating future changes with more people growing old and needing rehabilitation resources, there is considerable focus on structural changes of reimbursement and organization to improve the outcomes of Dutch GR within the limited financial resources. However, few data are available on the actual use of GR resources and outcomes. Therefore, this study aimed to identify basic patient and organizational characteristics of patients indicated for GR in SNFs related to outcomes of GR, such as length of stay (LOS) and discharge destination. In line with an acute care setting ${ }^{6,7}$ we hypothesized that a higher volume and/or concentration would be related to more successful GR in terms of shorter LOS and discharge home.

\section{Methods}

Design

This was a national multicenter retrospective cohort study in facilities providing GR.

The original goal of this study, ordered by Dutch Ministry of Health, was to estimate the total number of patients receiving GR in the Netherlands; therefore, all facilities providing GR were included. We used the data to answer our research questions (post hoc analysis).

\section{Recruitment of Facilities and Patients}

The Dutch government provided a list of all Dutch facilities providing GR ( $n=295)$. For each location, the institutional board of directors was informed of the study and invited to participate. During a 3-month period, in the participating facilities, all discharged patients who were indicated for GR after hospitalization were included. Exclusion criteria were primary diagnosis of dementia, not having an indication for GR, LOS in GR of 6 months or longer, and rehabilitation in a PAC setting but not in a GR ward on an SNF.

\section{Data Collection and Outcome Measures}

Data collection took place between October 2006 and October 2007. The participating facilities were randomly assigned to different 3-month periods.

\section{Outcomes}

The primary outcome was successful GR, defined as having a short LOS (total number of rehabilitation days in the facility) combined with being discharged to home (with or without day care). Short LOS was defined as the lowest 25\% LOS per diagnostic group. The outcome for patients who died in the SNF within the 3-month time frame was categorized as "not successful." Dutch SNFs are well equipped for palliative care and therefore it is unusual that a patient is sent home with the intention to die.

\section{Predictors}

The predictors in relation to the outcome "successful GR" were the organizational characteristics of "concentration" and "volume." "Concentration" was defined at the organizational level in which $80 \%$ or more of the included final population $(n=2269)$ undergoing GR in an SNF GR ward consists of 1 or 2 diagnostic groups. An additional prerequisite was that the facility should have a minimum of 10 rehabilitation beds. The few patients who were admitted to a concentrated ward with a diagnosis other than the concentration diagnoses of the ward were coded as not being on a concentrated ward.

For the definition of volume, we used the same procedure as described by $\mathrm{Li}$ et al. $^{8}$ Volume was based on the total population receiving GR in the GR ward. The facilities were categorized into tertile groups for volume: low volume ( $\leq 18$ discharges in a 3-month period), medium volume (19-28 discharges in 3-month period), and high volume (29-127 discharges in a 3-month period).

\section{Patient information}

Nurses filled out digital registration forms from patient records, including age, gender, medical diagnosis for GR, type of setting before GR and type of facility, LOS in GR, readmission rate to the hospital, and discharge destination. The medical diagnosis for GR was used to compose 3 diagnostic groups: total joint replacement, traumatic injuries, and stroke. In addition, a fourth group of patients was compiled with a mix of other medical diagnoses for GR (ie, "Other diagnosis for GR").

\section{Organization information}

The number of rehabilitation beds was obtained by structured telephone interview with the facility managers, or from a national database. These data were also obtained for the nonparticipating GR facilities.

The Medical Ethics Committee of the Leiden University Medical Center approved the study with a waiver of informed consent.

\section{Statistical Analysis}

Descriptive statistics were calculated for the characteristics of the population receiving GR in a GR ward of an SNF. Differences in characteristics between the patients in an SNF by type of medical diagnosis for GR were analyzed using a chi-square test. For age and LOS, differences were calculated with a Kruskal-Wallis test.

The generalized estimating equations (GEE) model was used to correct for cluster effects $(\mathrm{n}=88 \mathrm{SNFs}) .{ }^{16}$ Three GEE models (adjusted for age and gender) were applied for the 3 diagnostic groups (total joint replacement, trauma, and stroke) in relation to short LOS, discharge to home, and a final model with the combination of short LOS and discharge to home. Possible related organizational characteristics included volume and concentration. The diagnostic group "Other diagnosis for GR" was excluded because this group was very heterogeneous. Assumptions were checked for dependency within subjects and independency between subjects.

Sensitivity analyses were performed for seasonal effects and alternative definitions of volume groups (2 groups, quartile groups, and quintile groups). In the GEE model, an evaluation of effect modification was also performed with an interaction term (volume $x$ concentration). 


\section{Results}

Study Population

\section{Facilities}

Of the 295 GR facilities, 128 agreed to participate. Participating facilities did not differ from the nonparticipating facilities with regard to the number of beds and the geographical location (rural or urban) (data not shown).

There were 2 steps in the exclusion procedure of facilities. First, patients were excluded because of patient-related exclusion criteria, which led to the exclusion of 14 facilities and, second, patients who stayed in a PAC setting other than on an SNF GR ward were excluded, resulting in the exclusion of 26 facilities. Finally, 88 facilities remained for final analysis (Figure 1 ).

Of all facilities, $75 \%$ were not concentrated and had a heterogeneous population with several diagnostic groups receiving GR.

During a 3-month period, the 88 SNFs had a median volume of 24 admissions (interquartile range [IQR] 12.25-32, range 1 to 127).

\section{Patients}

Of the total 3371 patients, 256 were excluded: 67 with no rehabilitation indication, 53 with psychogeriatric diagnosis, 98 with LOS of 180 days or longer, 36 were duplicate cases, and 2 patients had incomplete data; also excluded were 846 patients who received rehabilitation in a PAC setting but not in a GR ward on an SNF (Figure 1). This resulted in a final study population of 2269 participants; mean age was 78.2 (SD 9.7) years and 68.2\% were women. In total, $22.1 \%$ had the primary diagnosis of total joint replacement
( $\mathrm{n}=502), 26.9 \%$ traumatic injury $(\mathrm{n}=611), 24.8 \%$ stroke $(\mathrm{n}=562)$, and $26.1 \%(n=594)$ had other GR indications. Most participants were admitted via a hospital (84.7\%).

Overall, the median LOS was 45 (IQR 23-81) days and $57 \%$ of the participants were discharged home. During the stay on the GR ward, 9.8\% died, with a considerable difference between the diagnostic groups (Table 1). The distribution of LOS also varied per diagnostic group: for trauma participants, the short LOS (and not deceased) was 35 days or less (25th percentile), for total joint replacement, short LOS was 11.25 days or less, and for stroke, it was 31.5 days or less. Of the total study population, $7.2 \%(\mathrm{n}=163)$ were readmitted to a hospital.

\section{Effects on Successful Geriatric Rehabilitation}

Table 2 shows the relationship (adjusted for gender and age) between organizational characteristics (volume and concentration) and the outcome of successful rehabilitation (short LOS and discharge home) for patients with total joint replacement $(n=501)$, trauma $(\mathrm{n}=611)$, and stroke $(\mathrm{n}=562)$.

In patients receiving total joint replacement, concentration was significantly related to the combined outcome of successful GR (odds ratio $[\mathrm{OR}] 5.67 ; 95 \%$ confidence interval $[\mathrm{CI}] 1.32-24.26 ; P=.020$ ). In the model with only short LOS and the model with only discharge home as the outcome, concentration was not significantly related to the combined outcome. Among patients receiving total joint replacement, volume was not related to successful rehabilitation (high volume OR 2.27; 95\% CI 0.50-10.18; $P=.286$; with low/medium volume as reference group). Because of low numbers, we combined low and medium volume as a reference group.

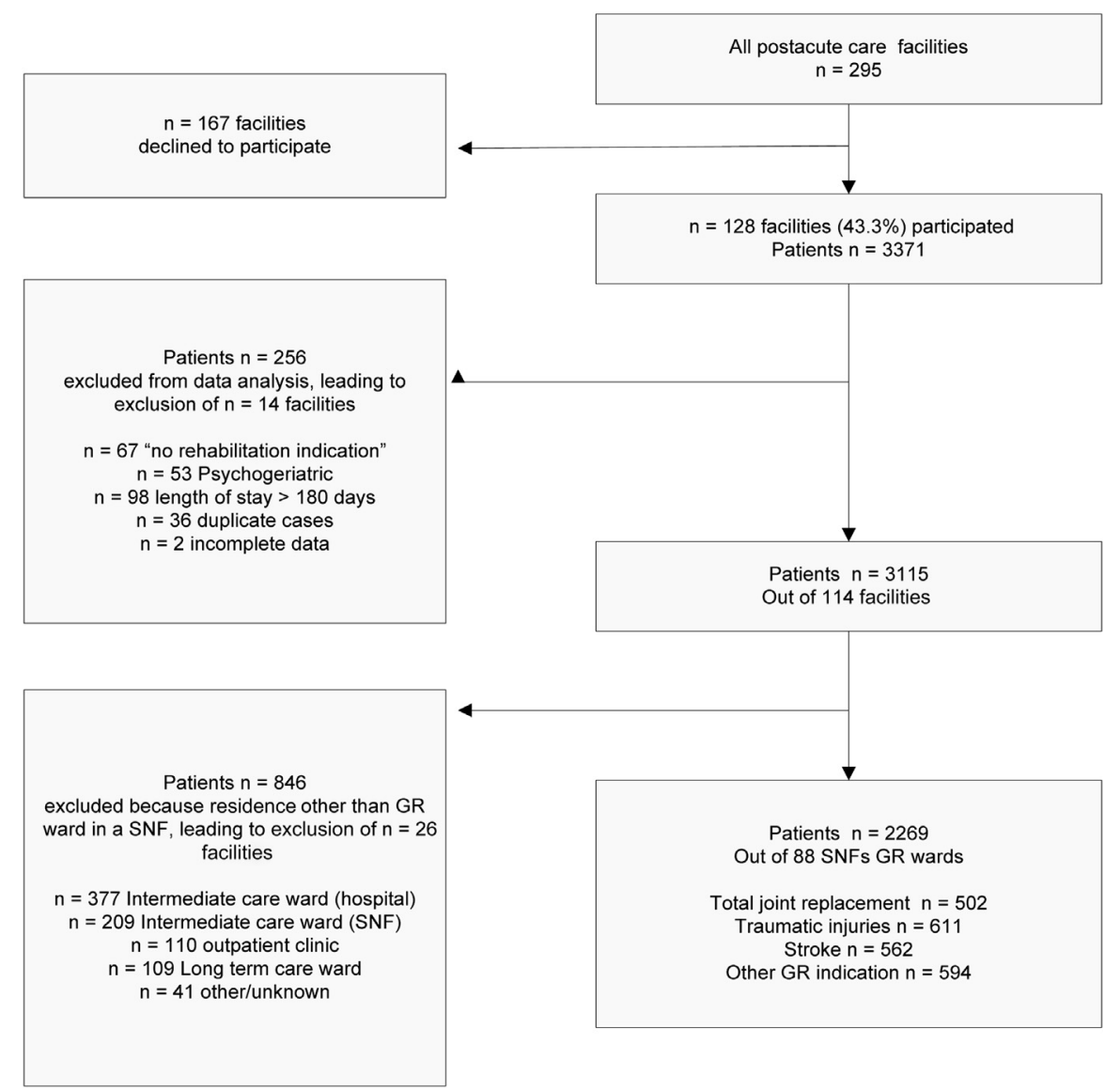

Fig. 1. Flowchart of the study, showing facilities and number of patients. 
Table 1

Characteristics of Patients in an SNF by Type of Medical Diagnosis for GR

\begin{tabular}{|c|c|c|c|c|c|c|}
\hline \multirow[t]{2}{*}{ Patient Characteristics } & \multirow{2}{*}{$\frac{\text { GR Ward (in SNF) }}{\mathrm{n}=2269}$} & \multirow{2}{*}{$\frac{\text { Total Joint Replacement }}{\mathrm{n}=502}$} & \multirow{2}{*}{$\frac{\text { Traumatic Injuries }}{\mathrm{n}=611}$} & \multirow{2}{*}{$\frac{\text { Stroke }}{\mathrm{n}=562}$} & \multirow{2}{*}{$\frac{\text { Other GR Indication }}{\mathrm{n}=594}$} & \multirow[t]{2}{*}{$P$ Value } \\
\hline & & & & & & \\
\hline \multicolumn{7}{|l|}{ Sex } \\
\hline Female & $1548(68.2)$ & $401(79.9)$ & $481(78.7)$ & $315(56.0)$ & $351(59.1)$ & $<.001$ \\
\hline Age, y & $78.2 \pm 9.7$ & $76.4 \pm 8.9$ & $79.5 \pm 10.4$ & $78.8 \pm 8.7$ & $77.8 \pm 10.3$ & $<.001$ \\
\hline \multicolumn{7}{|l|}{ Setting (before admission to PAC facility) } \\
\hline Hospital & $1922(84.7)$ & $447(89.0)$ & $527(86.3)$ & $497(88.4)$ & $451(75.9)$ & $<.001$ \\
\hline Living situation, home & $144(6.3)$ & $9(1.8)$ & $32(5.2)$ & $22(3.9)$ & $81(13.6)$ & \\
\hline Intermediate care & $94(4.2)$ & $33(6.6)$ & $21(3.4)$ & $25(4.4)$ & $15(2.5)$ & .008 \\
\hline Institutional residential care facility & $71(3.1)$ & $6(1.2)$ & $23(3.7)$ & $14(2.6)$ & $28(4.8)$ & .012 \\
\hline Rehabilitation center & $3(0.1)$ & $0(0.0)$ & $0(0.0)$ & $1(0.2)$ & $2(0.3)$ & $.384^{*}$ \\
\hline Other or unknown & $35(1.6)$ & $7(1.4)$ & $8(1.3)$ & $3(0.5)$ & $17(2.8)$ & $.031^{*}$ \\
\hline LOS median days (IQR) & $45(23-81)$ & $28(11.8-49)$ & $55(34-87)$ & $55(27.8-94)$ & $47(23-83.3)$ & $<.001$ \\
\hline \multicolumn{7}{|l|}{ Discharge location } \\
\hline Living situation, home & $1293(57.0)$ & $423(84.2)$ & $394(64.5)$ & $215(38.3)$ & $261(43.9)$ & $<.001$ \\
\hline Home with outpatient day care and treatment & $67(3.0)$ & $4(0.8)$ & $13(2.1)$ & $35(6.2)$ & $15(2.5)$ & $<.001^{*}$ \\
\hline Hospital & $163(7.2)$ & $23(4.6)$ & $40(6.5)$ & $30(5.3)$ & $70(11.8)$ & $<.001$ \\
\hline Rehabilitation center & $9(0.4)$ & $0(0.0)$ & $1(0.2)$ & $7(1.2)$ & $1(0.2)$ & $.0016^{*}$ \\
\hline Institutional residential care facility & $457(20.1)$ & $32(6.4)$ & $111(18.1)$ & $185(33.0)$ & $129(21.7)$ & $<.001$ \\
\hline Other or unknown & $57(2.5)$ & $14(2.8)$ & $21(3.5)$ & $8(1.4)$ & $14(2.4)$ & .329 \\
\hline Deceased & $223(9.8)$ & $6(1.2)$ & $31(5.1)$ & $82(14.6)$ & $104(17.5)$ & $<.001$ \\
\hline
\end{tabular}

GR, geriatric rehabilitation; IQR, interquartile range; LOS, length of stay; PAC, postacute care facility; SNF, skilled nursing facility.

Values are numbers $(\%)$ or mean \pm SD unless indicated otherwise. $P$ value was calculated with $\chi^{2}$ test, except for age and LOS calculated with Kruskal-Wallis test.

*Fisher's exact test.

In patients with traumatic injuries, concentration was significantly related to discharge home (OR 1.89; CI 1.14-3.11; $P=.013$ ); however, the combined outcome for successful rehabilitation showed no significant differences.

In patients with stroke, neither volume nor concentration had a significant relation to the outcome of successful rehabilitation.

Results of the sensitivity analysis on seasonal influences and on alternative definitions of volume did not alter the effect estimators of the final model (data not shown). In the final model, there was no interaction of volume and concentration within the 3 diagnostic groups.

\section{Discussion}

This is the first study to describe GR within a large sample of GR facilities in the Netherlands. In this national multicenter retrospective cohort study of patients indicated for GR in an SNF, $75 \%$ of the facilities had a heterogeneous population with different diagnostic groups receiving GR. During a 3-month period, the 88 SNFs had a median volume of 24 (IQR 12.25-32) discharges.

High concentration, but not volume, was related to successful rehabilitation only in the total joint replacement group. Further examination of the reasons for this benefit is important when redesigning or improving the organization of GR for these patients.

The concentrated GR facilities performed better in the total joint replacement group for the outcome of successful rehabilitation. For patients with traumatic injuries, a significant relation was found in the model with only the outcome of discharge home. No relationship with concentration was found in the group of patients with stroke. Earlier studies showed some efficacy with concentrated stroke units in an inpatient facility, although high-quality RCTs are still lacking for

Table 2

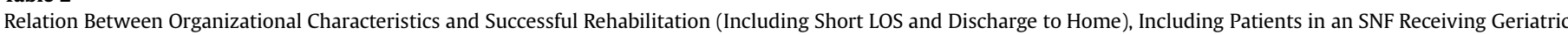
Rehabilitation With the Diagnosis of Traumatic Injuries, Stroke, and Total Joint Replacement $(\mathrm{n}=1674)$

\begin{tabular}{|c|c|c|c|c|c|c|c|}
\hline & \multicolumn{4}{|c|}{ Volume } & \multicolumn{3}{|c|}{ Concentration } \\
\hline & \multirow{2}{*}{$\frac{\text { Low }^{*}}{\text { Ref }}$} & \multirow{2}{*}{$\frac{\text { Medium }^{\dagger}}{\text { OR }(95 \% \mathrm{CI})}$} & \multirow{2}{*}{$\frac{\operatorname{High}^{\ddagger}}{\text { OR }(95 \% \mathrm{CI})}$} & \multirow[t]{2}{*}{$P$ Trend $/ P$ Value $^{\S}$} & \multirow{2}{*}{$\frac{\text { No }}{\text { Ref }}$} & \multirow{2}{*}{$\frac{\text { Yes }}{\text { OR }(95 \% \mathrm{CI})}$} & \multirow[t]{2}{*}{$P$ Value } \\
\hline & & & & & & & \\
\hline \multicolumn{8}{|l|}{ Traumatic injuries $(\mathrm{n}=611)$} \\
\hline Short LOS & 1 & $0.67(0.35-1.31)$ & $1.07(0.58-1.99)$ & .271 & 1 & $0.93(0.60-1.43)$ & .730 \\
\hline Discharge home & 1 & $0.78(0.41-1.49)$ & $0.77(0.41-1.45)$ & .488 & 1 & $1.89(1.14-3.11)$ & .013 \\
\hline Short LOS + Discharge home & 1 & $0.81(0.37-1.78)$ & $0.95(0.45-2.01)$ & .885 & 1 & $1.02(0.58-1.78)$ & .945 \\
\hline \multicolumn{8}{|l|}{ Stroke $(\mathrm{n}=562)$} \\
\hline Short LOS & 1 & $0.71(0.35-1.42)$ & $1.00(0.51-1.98)$ & .718 & 1 & $1.26(0.72-2.24)$ & .421 \\
\hline Discharge home & 1 & $1.03(0.56-1.92)$ & $0.81(0.43-1.51)$ & .382 & 1 & $0.67(0.39-1.14)$ & .141 \\
\hline Short LOS + Discharge home & 1 & $0.68(0.31-1.49)$ & $0.83(0.39-1.81)$ & .862 & 1 & $0.82(0.41-1.64)$ & .568 \\
\hline \multicolumn{8}{|l|}{ Total joint replacement $(\mathrm{n}=501)$} \\
\hline Short LOS $^{\#}$ & $1^{* *}$ & & $2.80(0.70-11.23)$ & .147 & 1 & $3.48(0.92-13.19)$ & .067 \\
\hline Discharge home & $1^{* *}$ & & $0.51(0.25-1.07)$ & .075 & 1 & $2.10(0.89-4.96)$ & .089 \\
\hline Short LOS + Discharge home & $1^{* *}$ & & $2.27(0.50-10.18)$ & .286 & 1 & $5.67(1.32-24.26)$ & .020 \\
\hline
\end{tabular}

$\mathrm{CI}$, confidence interval; LOS, length of stay; OR, odds ratio; Ref, reference group, SNF, skilled nursing facility.

ORs were estimated by generalized estimating equations adjusted for gender and age.

*29-127 discharges during a 3-month period.

${ }^{\dagger} 19-28$ discharges during a 3-month period.

${ }^{\ddagger} \leq 18$ discharges during a 3-month period.

${ }^{\S} P$ trend was calculated in case of $>2$ volume groups and in case of 2 volume groups $P$ value was calculated.

$\| \leq 35$ days.

$\pi \leq 31.5$ days.

${ }^{\#} \leq 11.25$ days.

** Because of low numbers, low and medium volume were combined as a reference group for the diagnosis group total joint replacement. 
units especially designed for geriatric patients. ${ }^{11,12}$ However, patients in an inpatient rehabilitation facility may differ from the population in an SNF (ie, they are usually younger and have a better functional prognosis compared with patients indicated for GR in an SNF). ${ }^{12}$

Volume had no significant effect on the outcome of successful rehabilitation. For the group with joint replacement, the ORs suggest that a high volume results in more successful GR; however, this result was not significant and was mainly due to a short LOS. Also, total joint replacement is generally planned care and the continuous care chain might be better organized in these patients compared with patients with traumatic injuries and/or stroke. However, this can also lead to selection bias in which high-volume PAC settings select patients with a good prognostic outcome resulting in a short LOS. Also, in acute care, the results are difficult to compare because of different levels of analysis (eg, hospital, surgeon, ward, or patient level) and different outcome measures. ${ }^{17}$

In most studies, rehospitalization rate is another important outcome indicator for SNF care quality. ${ }^{8,18}$ In the present study, the rehospitalization rate was relatively low (7.2\%) compared with PAC facilities in the United States, with an estimated rate of $23.5 \%$ within 30 days. ${ }^{19}$ We are not sure whether this is the effect of an intensive interdisciplinary approach and/or the presence of a trained elderly care physician leading to a higher quality of medical care within Dutch SNFs, or whether this reflects case-mix differences. ${ }^{18}$ However, the rate is in line with other studies showing low hospital referral rates in long term care facilities in the Netherlands. ${ }^{20}$

Researchers and policymakers worldwide are focusing on the effect of volume and concentration of treatment/care to improve cost-effectiveness. ${ }^{6,8,10,14,21}$ Concentration suggests a level of specialization within the organization. Therefore, in our definition of concentration, we combined the predominance of 1 or 2 diagnostic groups with a minimum of 10 rehabilitation beds, to meet the conditions of specialization. Facilities with a concentration on specific diagnostic groups are able to form a specialized multidisciplinary team, operate more efficiently, and develop more skills, possibly resulting in better outcomes ("practice makes perfect effect"). In turn, facilities with a good reputation will attract more patients, resulting in a higher volume ("selective referral effect"), and these 2 effects interact with each other. 6,8 Thus, concentration as a proxy for specialization could be a predictor for better outcomes. However, in GR it is unknown which characteristics the most optimal organization should have in daily practice, and which type of patient is best suited for which rehabilitation path. ${ }^{22}$ In the present study, our definition of concentration may not be fully equivalent to an efficiently operating multidisciplinary specialized team. For instance, we lacked data on the level of expertise/years of experience of the individual team members, which may have influenced the results. ${ }^{17}$ Future research should explore the active ingredients of concentration, what best reflects the quality of care, and how organizational characteristics might improve functional outcomes.

Because this was a national retrospective study, only limited data were available; we lacked potentially interesting data on (for example) individual functional outcomes, functional level before admission, length of hospital stay, living location before hospitalization, and the active ingredients of concentration. Also, power was restricted for further subgroup analysis. Future studies exploring the effect of geriatric revalidation need to include these data, which are also related to outcomes of GR. Despite these limitations, this is the first study to describe the total population in the Netherlands receiving GR, with a large sample of all Dutch PAC facilities providing GR. Another strength is that we used the GEE model to adjust for correlated observations within the SNF clusters, which other studies often lacked. Cluster correlation can lead to an overestimation of the effect. $^{16}$

\section{Conclusion}

This study may indicate that concentration in an SNF, as a proxy for specialization, within the diagnostic group total joint replacement favors successful GR, because concentration of the GR facility was related to a shorter LOS and more discharge to home. For the other diagnostic groups, and for the characteristic volume, no such relationship was found. Future research needs to explore the relation on functional outcome in GR.

\section{Acknowledgments}

The authors thank ETC-TANGRAM for data collection.

\section{References}

1. United Nations. Department of Economic and Social Affairs. Population Division. Available at: http://www.un.org/esa/population/. Accessed August 26, 2011.

2. Buurman BM, Hoogerduijn JG, de Haan RJ, et al. Geriatric conditions in acutely hospitalized older patients: Prevalence and one-year survival and functional decline. PLoS One 2011;6:e26951.

3. de Saint-Hubert M, Schoevaerdts D, Poulain G, et al. Risk factors predicting later functional decline in older hospitalized patients. Acta Clin Belg 2009;64:187-194.

4. Stucki G, Stier-Jarmer M, Grill E, Melvin J. Rationale and principles of early rehabilitation care after an acute injury or illness. Disabil Rehabil 2005;27: 353-359.

5. Boston Working Group on Improving Health Care Outcomes Through Geriatric Rehabilitation. Med Care 1997;35:JS4-JS20.

6. Halm EA, Lee C, Chassin MR. Is volume related to outcome in health care? A systematic review and methodologic critique of the literature. Ann Intern Med 2002;137:511-520.

7. Shahin J, Harrison DA, Rowan KM. Relation between volume and outcome for patients with severe sepsis in United Kingdom: retrospective cohort study. BMJ 2012;344:e3394.

8. Li Y, Cai X, Yin J, et al. Is higher volume of postacute care patients associated with a lower rehospitalization rate in skilled nursing facilities? Med Care Res Rev 2012;69:103-118.

9. Gindin J, Walter-Ginzburg A, Geitzen M, et al. Predictors of rehabilitation outcomes: a comparison of Israeli and Italian geriatric post-acute care (PAC) facilities using the minimum data set (MDS). J Am Med Dir Assoc 2007;8: $233-242$.

10. Rimar JM, Diers D. Inpatient nursing unit volume, length of stay, cost, and mortality. Nurs Econ 2006;24:298-307. 279.

11. Bachmann S, Finger $C$, Huss $A$, et al. Inpatient rehabilitation specifically designed for geriatric patients: Systematic review and meta-analysis of randomised controlled trials. BMJ 2010;340:c1718.

12. Prvu Bettger JA, Stineman MG. Effectiveness of multidisciplinary rehabilitation services in postacute care: State-of-the-science. A review. Arch Phys Med Rehabil 2007;88:1526-1534.

13. Langhorne P, Bernhardt J, Kwakkel G. Stroke rehabilitation. Lancet 2011;377: 1693-1702.

14. Tian W, DeJong G, Horn SD, et al. Efficient rehabilitation care for joint replacement patients: Skilled nursing facility or inpatient rehabilitation facility? Med Decis Making 2012;32:176-187.

15. Koopmans RT, Lavrijsen JC, Hoek JF, et al. Dutch elderly care physician: A new generation of nursing home physician specialists. J Am Geriatr Soc 2010;58: 1807-1809.

16. French B, Farjah F, Flum DR, Heagerty PJ. A general framework for estimating volume-outcome associations from longitudinal data. Stat Med 2012;31: 366-382.

17. Christian CK, Gustafson ML, Betensky RA, et al. The volume-outcome relationship: Don't believe everything you see. World J Surg 2005;29: 1241-1244.

18. Dombrowski W, Yoos JL, Neufeld R, Tarshish CY. Factors predicting rehospitalization of elderly patients in a postacute skilled nursing facility rehabilitation program. Arch Phys Med Rehabil 2012;93:1808-1813.

19. Mor V, Intrator O, Feng Z, Grabowski DC. The revolving door of rehospitalization from skilled nursing facilities. Health Aff (Millwood) 2010;29:57-64.

20. van der Steen JT, Kruse RL, Ooms ME, et al. Treatment of nursing home residents with dementia and lower respiratory tract infection in the United States and The Netherlands: An ocean apart. J Am Geriatr Soc 2004;52:691-699.

21. Li Y, Cai X, Mukamel DB, Glance LG. The volume-outcome relationship in nursing home care: An examination of functional decline among long-term care residents. Med Care 2010;48:52-57.

22. Heinemann AW. State of the science on postacute rehabilitation: Setting a research agenda and developing an evidence base for practice and public policy. An introduction. Assist Technol 2008;20:55-60. 